\title{
Lug Metal Container Closure
}

National Cancer Institute

\section{Source}

National Cancer Institute. Lug Metal Container Closure. NCI Thesaurus. Code C96126.

Closure with an ability to be applied and removed with a partial turn. The closure can also be produced with vacuum buttons that can clearly indicate to the packer if a vacuum has been effectively drawn following the closure application. 